\title{
Inferência dos Perfis de Infusão em Sistemas Intravenosos: Uma Abordagem Empregando Técnicas de Aprendizagem de Máquina
}

\begin{abstract}
Fabrício Ferreira $^{1}$, Felipe Gruendemann ${ }^{1}$, Ricardo Araujo ${ }^{1}$, Adenauer Yamin $^{1}$, Luciano Agostini $^{1}$

${ }^{1}$ Universidade Federal de Pelotas (UFPel) - Pelotas - RS - Brasil

Email:\{fferreira,fcgruendemann,ricardo,adenauer,agostini\}@inf.ufpel.edu.br

Abstract. Intravenous infusion procedures are present when rapid therapeutic return is required. These infusion procedures are among the most usually in hospitals and have the potential to generate a high volume of adverse events. However, infusions still have their inspection perform out based on checked non-automatically, thus, they are subject to failures of different natures. Considering this scenario, this article presents an evaluation of six regression methods used in Machine Learning and applied in the automatic identification of different profiles of intravenous infusions. The evaluations performed out to show that regression models by neural networks are promising for the inferences of infusions.
\end{abstract}

Resumo. Os procedimentos de infusão intravenosa estão entre os mais usuais em hospitais e têm potencial para gerar alta ocorrência de eventos adversos. No entanto, as infusões intravenosas ainda não têm a sua verificação automatizada. Considerando este cenário, este trabalho propõe uma nova abordagem para reduzir eventos adversos em procedimentos intravenosos utilizando Aprendizado de Máquina para permitir uma inferência autônoma e registro dos perfis de infusões intravenosas. Dois regressores baseados em redes neurais foram avaliados: Multi-Layer Perceptron e Long-Short Term Memory. A avaliação dos modelos regressão, para as inferências dos perfis de administração de medicamentos intravenosos, obtiveram resultados promissores.

\section{Introdução}

Os sistemas intravenosos são usados na maioria dos procedimentos clínicos de rotina em hospitais e estima-se que cerca de $80 \%$ dos pacientes hospitalizados necessitem desses recursos terapêuticos [Sait et al. 2019].

Em relação aos riscos associados ao uso de tecnologias em saúde, o Emergency Care Research Institute (ECRI), que anualmente classifica os 10 maiores riscos de uso de tecnologias no ambiente hospitalar, identificou que os erros dos dispositivos intravenosos foram classificados em 2017 como os primeiros neste ranking [ECRI 2017, Dos Santos et al. 2019].

Considerando que o uso dos dispositivos intravenosos ocorrem em situações onde os pacientes se encontram em situação de saúde vulnerável, esses erros na entrega de medicamentos intravenosos podem ter impactos severos no tratamento de um paciente [Chambers 2019]. 
Logo, é um grande desafio tecnológico para os sistemas de apoio à decisão, embarcados nos dispositivos de infusão intravenosa, obter precisão na entrega de medicamentos, visto que sua composição agrega múltiplos componentes, de diferentes fabricantes [NBR 60601-2-24 2015, ECRI 2017]. Os principais itens de dispositivos intravenosos que influenciam a administração de medicamentos são: (i) tubos de infusão; (ii) reservatório de droga e (iii) bombas de infusão [Chambers 2019].

As infusões intravenosas são geralmente monitoradas por paramédicos e, portanto, estão sujeitas às falhas inerentes a um acompanhamento feito de forma manual. É importante observar que qualquer negligência em procedimentos intravenosos tem um alto potencial de risco para a vida do paciente [Fink et al. 2018].

Diante desse cenário, dois aspectos constituem a motivação central deste trabalho: (i) os procedimentos terapêuticos intravenosos são atualmente a atividade clínica mais popular e usual em hospitais em todo o mundo [Sait et al. 2019]; (ii) por outro lado, são praticados sem controle automatizado, o que aumenta os riscos associados ao não atendimento das prescrições médicas no que diz respeito a entrega de medicamentos infundidos [Chambers 2019].

Com base nesta motivação, o objetivo principal deste trabalho é o desenvolvimento de método para identificar os perfis de infusões intravenosas de forma autônoma, a partir de dados obtidos por sensoriamento. A inferência precisa dos perfis das entregas de medicamentos intravenosos é importante para: (i) permitir que os profissionais de saúde observem rapidamente algum comportamento inesperado no processo de infusão, evitando riscos causados por uma vazão de infusão incorreta; e (ii) permitir o registro automático da infusão realizada, o que é importante para que os profissionais de saúde avaliem os efeitos terapêuticos do medicamento infundido. Nesse sentido, este trabalho explorou os dados gerados por um simulador de perfil de infusão de dispositivo intravenoso, discutido pelos autores em publicações anteriores [Ferreira et al. 2019].

Este simulador permite a geração ágil de diferentes perfis de infusão para dispositivos intravenosos gravitacionais e bomba de infusão, permitindo o desenvolvimento de experimentos sem a necessidade de medições de dados reais ${ }^{1}$.

Logo, as principais contribuições deste trabalho são uma proposta de solução autônoma baseada em aprendizado de máquina para identificar os perfis das entregas de medicamentos dos dispositivos intravenosos por infusão gravitacional e por bomba de infusão. Tendo, como resultado uma avaliação de dois modelos de inferência de regressão, MultiLayer Perceptron (MLP) e Long-Short Term Memory (LSTM), explorando um conjunto, com dados simulados e reais, contemplando características usuais de infusões intravenosas.

\section{Metodologia Proposta para Inferir Perfis Intravenosos}

A abordagem metodológica proposta neste trabalho baseia-se na ideia de que um perfil de infusão intravenosa regular pode ser comparado com perfis de infusões previamente registradas para inferir qual é a vazão da infusão atual e, com essas informações, identificar se a infusão se comporta corretamente durante o período que está acontecendo.

\footnotetext{
${ }^{1} \mathrm{O}$ simulador e o conjunto de dados simulados usados neste trabalho, estão disponíveis para o público em [NEITZKE F. 2020b, NEITZKE F. 2020a]
} 
O Conjunto de Dados, explorado neste trabalho, está organizado em características rotuladas, que caracterizam o sistema intravenoso como um todo: (i) o volume do medicamento administrado; (ii) o valor da taxa de vazão; (iii) o tipo de dispositivo intravenoso; e (iv) o registro temporal das informações. Assim, considerando que esses dados estão rotulados, o método de aprendizado de máquina oportuno para construção de um modelo para sistemas intravenosos é o supervisionado [Hao and Ho 2019].

O modelo de inferência desenvolvido para os perfis de vazão considerou infusões intravenosas de diferentes volumes nominais do reservatório do medicamento: [125, 250, 500 e 1000] $m L$. Esses volumes foram usados tanto para modelar infusões gravitacionais, como infusões empregando bombas.

O conjunto de dados disponível compreende mais de 3.793 horas de infusões intravenosas, totalizando 13.655 .400 amostras e 829 tipos de perfis com diferentes taxas de vazão. Conforme discutido anteriormente, este conjunto de dados foi gerado usando um simulador de perfil de infusão. Este simulador utilizou modelos de dados de sensores de células de carga como base para gerar os perfis de infusão. Nesse caso, o simulador considera que o reservatório do fármaco está sustentado por um sensor de célula de carga cujas saídas são utilizadas para instrumentar os perfis das infusões. As saídas da célula de carga são valores de tensão elétrica proporcionais ao peso da força exercida pelo reservatório de medicamento. Então, é possível inferir o comportamento do perfil das infusões intravenosas usando essas informações da tensão elétrica. Esta abordagem é amplamente aceita na comunidade científica que explora a flutuação de peso para inferir o volume entregue [Ferreira et al. 2019, Bhavasaar et al. 2016].

Assim, as saídas do simulador correspondem aos valores amostrados da célula de carga durante a janela de tempo de infusão, logo as saídas do simulador são um grupo de valores em volts. O usuário pode definir a taxa de amostragem e a janela de tempo registrada.

A Tabela 1 especifica a quantidade de dados que compõem o conjunto de dados do perfil de infusão usado neste trabalho, organizado pelo volume do reservatório de medicamentos.

Tabela 1. Informação do Conjunto de Dados dos Perfis de Infusão

\begin{tabular}{c|c|c|c}
\hline Volume do & \multicolumn{2}{|c|}{ Número de Perfis de Infusão } & Infusão \\
\cline { 2 - 3 } Reservatório $[\mathrm{mL}]$ & Infusão Gravitacional & Bombas de Infusão & [Horas] \\
\hline 125 & 190 & 374 & 611 \\
\hline 250 & 90 & 90 & 284 \\
\hline 500 & 180 & 180 & 1.098 \\
\hline 1000 & 180 & 320 & 1.779 \\
\hline
\end{tabular}

Já a Tabela 2 mostra informações parciais sobre os dados, Atributos e Alvos, para cada tipo de dispositivo de infusão. Onde os dados são registros do sensoriamento que representa o comportamento do perfil de infusão para uma sequência de Janela Temporal de 60 segundos. Os rótulos das colunas Atributos são: $T e m p_{0}$ que indica o início da Janela Temporal e $T e m p_{59}$ é o fim dessa Janela Temporal. Os outros rótulos (Temp $p_{1}$ a $\left.T e m p_{58}\right)$ foram omitidos para simplificar a apresentação. Os Alvos são definidos pelo rótulo da coluna Taxa, configurando a velocidade do perfil da taxa de entrega de medicamento in- 


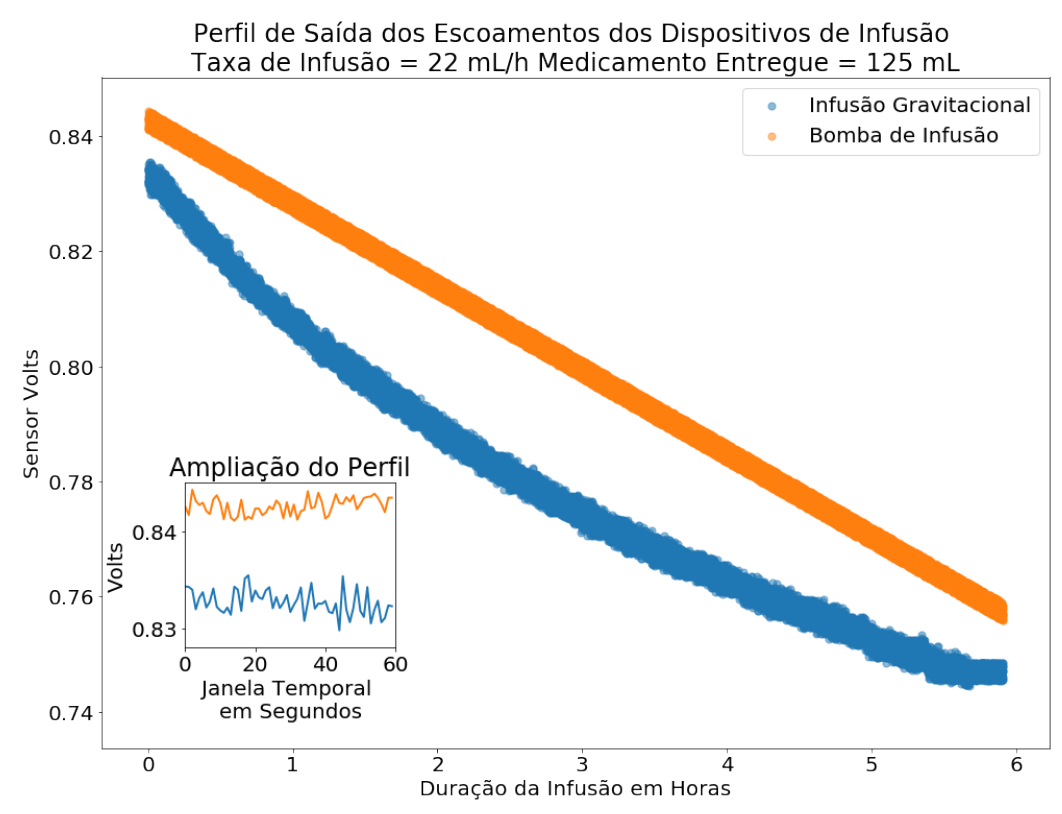

Figura 1. Perfil de Infusão dos Dispositivos Intravenosos.

travenosa em $m L / h$, para os diferentes perfis de intravenosos registrados.

Além disso, na Tabela 2, na coluna Especificação, é informado o volume do medicamento com a distinção entre os valores nominais e o volume entregue. Essas diferenças entre os volumes de medicamentos são típicas em sistemas intravenosos [Chambers 2019]. A coluna Especificação não foi considerada como um atributo para o desenvolvimento da modelagem e apenas as colunas Atributos e Taxa foram consideradas. Por fim, a Tabela 2 apresenta, para cada Janela Temporal, os valores em volts produzidos pelo simulador considerando um sensor de célula de carga, conforme discutido anteriormente.

Tabela 2. Conjunto de Dados Explorado na Aprendizagem de Máquina

\begin{tabular}{|c|c|c|c|c|c|c|c|}
\hline \multicolumn{3}{|c|}{ Infusão Gravitacional } & \multicolumn{3}{|c|}{ Bombas de Infusão } & \multirow{2}{*}{\multicolumn{2}{|c|}{$\begin{array}{l}\text { Especificação } \\
\text { Volume [mL] }\end{array}$}} \\
\hline Atribut & [Volts] & Alvos $[\mathrm{mL} / \mathrm{h}]$ & Atribut & [Volts] & Alvo [mL/h] & & \\
\hline Temp $_{0}$ & Temp $_{59}$ & Taxa & Temp $_{0}$ & Temp $_{59}$ & Taxa & Entregue & Nominal \\
\hline 0,83451 & 0,83221 & 22,0 & 0,83531 & 0,83666 & 22,0 & 124,6 & 125,0 \\
\hline 0,83157 & 0,83157 & 22,0 & 0,83570 & 0,83542 & 22,0 & 124,6 & 125,0 \\
\hline 0,83140 & 0,83021 & 22,0 & 0,83416 & 0,83570 & 22,0 & 124,6 & 125,0 \\
\hline
\end{tabular}

A Figura 1 apresenta os gráficos de um exemplo dos perfis de administração de medicamentos para ambos os dispositivos de infusão intravenosa gerados com o simulador usado [Ferreira et al. 2019]. Este exemplo mostra os perfis de infusão apresentando uma taxa de vazão de $22 \mathrm{~mL} / \mathrm{h}$, com uma entrega total de $125 \mathrm{~mL}$ de medicamento. Na Figura 1, é detalhado uma ampliação dos perfis de infusão, cujo tamanho da amostra é igual à Janela Temporal, portanto, com um intervalo de 60 segundos de duração.

Na Figura 1, o eixo Y corresponde ao sinal elétrico em Volts produzido pelo sensor de vazão do líquido medicado. Ao mesmo tempo, o eixo $\mathrm{X}$ indica o avanço do tempo em horas de infusão. Na Janela Temporal destacada na Figura 1, a unidade de tempo do eixo 
X é em segundos.

\subsection{Modelagem dos Perfis de Infusão Intravenosos}

Na exploração dos métodos de modelagem dos regressores MLP e LSTM, foram exploradas as ferramentas de aprendizado de máquina das bibliotecas Scikit-Learn e Pytorch, como um método para as inferências das entregas de medicamentos intravenosos [Pedregosa et al. 2011, Paszke et al. 2019]. A seleção dessas bibliotecas foi motivada por: (i) serem exploradas em diversas aplicações de pesquisas acadêmicas; (ii) possuem as implementações dos métodos explorados neste trabalho; e (iii) são de código aberto [Hao and Ho 2019].

Os dados simulados dos perfis das infusões intravenosas foram organizados em duas partes, destinadas respectivamente às duas etapas de construção do modelo: Treinamento e Teste. A Tabela 3 mostra a separação dos dados; essas variações de porcentagens da quantidade de dados manipulados nas fases de Treinamento e Teste são tipicamente exploradas no processo de obtenção de modelagem por Aprendizado de Máquina.

Tabela 3. Conjunto de Dados Simulados das Etapas de Treinamento e Teste

\begin{tabular}{c|c|c|c|c|c|c}
\hline Dispositivo & \multicolumn{2}{|c|}{ Número de Amostra } & \multicolumn{2}{c|}{ Infusão [Horas] } & \multicolumn{2}{c}{ Porcentagem [\%] } \\
\cline { 2 - 7 } de Infusão & Treinamento & Teste & Treinamento & Teste & Treinamento & Teste \\
\hline Gravitacional & 5.458 .920 & 1.052 .100 & 1.516 & 292 & 82 & 18 \\
\hline Bomba & 5.906 .220 & 1.238 .160 & 1.640 & 343 & 83 & 17 \\
\hline
\end{tabular}

A seleção dos dados das etapas de construção da modelagem e sua avaliação foi aleatória e sempre considerou os dados Alvos (Taxa) com a infusão completa. Portanto, esse método de seleção não permitiu a segmentação dos dados entre as etapas de Treinamento e Teste. Esta decisão contribui para evitar o sobre-ajuste [Hao and Ho 2019].

\subsection{Avaliações do Modelo de Regressão}

Esta sessão apresenta as avaliações dos métodos de Aprendizagem de Máquina utilizados neste trabalho. Foram avaliados os modelos produzidos com a utilização dos regressores MLP e LSTM na inferência dos perfis de infusão intravenosa. Infusões gravitacionais e por bombas de infusão foram modeladas usando essa estratégia.

A construção dos modelos explorou diferentes camadas de arquiteturas e o ajuste dos hiper-parâmetros para os regressores MLP e LSTM com o objetivo de obter a melhor modelagem possível dos perfis de infusão intravenosa. Assim, diferentes camadas de arquiteturas e hiper-parâmetros foram avaliados, considerando as recomendações das ferramentas utilizadas [Pedregosa et al. 2011, Paszke et al. 2019].

A Tabela 4 descreve as combinações das camadas arquiteturas e os parâmetros avaliados na busca pelas melhores métricas para o modelo de regressão MLP, que foi implementado com as ferramentas Scikit-Learn [Pedregosa et al. 2011]. Por sua vez, a Tabela 5 descreve as combinações das camadas de arquitetura e os parâmetros avaliados na busca pelas melhores métricas para o modelo de regressão LSTM, que foi implementado com a ferramenta Pytorch [Paszke et al. 2019].

As melhores combinações de parâmetros definidas para o modelo de regressão MLP são mostradas na Tabela 6. 
Tabela 4. Combinações das Configurações para o Regressor MLP

\begin{tabular}{c|c|c}
\hline Parâmetros & \multicolumn{2}{|c}{ Multi-Layer Perceptron } \\
\hline Hidden & Neurons & Hidden Layer \\
\cline { 2 - 3 } Layer Sizes & {$[10,50,100,200,300]$} & {$[1,2,10,50]$} \\
\hline Activation & ['identity', 'logistic', 'tanh', 'relu'] \\
\hline Solver & \multicolumn{2}{|c}{ ['lbfgs', 'adam'] } \\
\hline Alpha & \multicolumn{2}{|c}{$[0.0001,0.01,0.1]$} \\
\hline Max_iter & \multicolumn{2}{|c}{$[200,2000]$} \\
\hline
\end{tabular}

Tabela 5. Combinações das Configurações para o Regressor LSTM

\begin{tabular}{c|c}
\hline Parâmetros & Long-Short Term Memory \\
\hline Input_dim & {$[1,60]$} \\
\hline Batch_size & {$[1,120,240]$} \\
\hline Hidden_dim & {$[60,120,180,240,300,420]$} \\
\hline Loss Function & {$[$ L1Loss, MSELoss $]$} \\
\hline Optimizer & {$[$ RMSprop, Adam, SGD $]$} \\
\hline Lstm_out & {$[$ linear $]$} \\
\hline Num_layers & {$[2,3,4,6]$} \\
\hline Epoch & {$[10000,20000]$} \\
\hline
\end{tabular}

Tabela 6. Melhores Combinações para o Regressor MLP

\begin{tabular}{c|c|c|c|c|c}
\hline Dispositivo de Infusão & Hidden, Layer Sizes & Activation & Solver & Alpha & Max_iter \\
\hline Gravitacional & $(200,10)$ & tanh & adam & 0.1 & 200 \\
\hline Bomba & $(50,10)$ & relu & adam & 0.1 & 2000 \\
\hline
\end{tabular}

Com relação ao modelo de regressão LSTM, a Tabela 7 mostra as combinações dos melhores parâmetros selecionados. O regressor LSTM usou a função de perda do erro quadrático médio com uma redução pela média [Paszke et al. 2019].

Tabela 7. Melhores Combinações para o Regressor LSTM

\begin{tabular}{c|c|c|c|c|c|c}
\hline $\begin{array}{c}\text { Dispositivo } \\
\text { de Infusão }\end{array}$ & $\begin{array}{c}\text { Input_dim } \\
\text { Batch_size } \\
\text { Hidden_dim }\end{array}$ & $\begin{array}{c}\text { Loss } \\
\text { Function }\end{array}$ & $\begin{array}{c}\text { Lstm } \\
\text { Out }\end{array}$ & $\begin{array}{c}\text { Nun } \\
\text { Layers }\end{array}$ & Optimizer & Epoch \\
\hline Gravitacional & $(60,1,240)$ & MSELoss & Linear & 2 & RMSprop & 20000 \\
\hline Bomba & $(60,1,240)$ & MSELoss & Linear & 4 & RMSprop & 20000 \\
\hline
\end{tabular}

\section{Resultados}

A análise dos resultados avaliou a concepção da modelagem com dados simulados com os perfis intravenosos inferidos. Também avaliou a qualidade das inferências de regressão LSTM, com dados reais dos perfis intravenosos, dados nunca manipulados antes.

\subsection{Resultados da Avaliação Explorando o Conjunto de Dados Simulados}

$\mathrm{Na}$ construção dos modelos de regressor foram utilizados 1.560 perfis de entrega de medicamentos intravenosos explorando dispositivos de infusão gravitacional e bombas de 
infusão. A métrica Erro Médio Absoluto (MAE) foi adotada para avaliar a precisão dos modelos MLP e LSTM [Pedregosa et al. 2011].

A Tabela 8 apresenta os resultados do MAE para ambos os modelos de regressão e os dois dispositivos de infusão intravenosa, organizados pelo volume do reservatório.

Tabela 8. Avaliação dos Regressores na Etapa de Teste com Dados Simulados

\begin{tabular}{c|c|c|c}
\hline \multirow{2}{*}{$\begin{array}{c}\text { Volume do Reservatório } \\
\text { de Medicamento }[m L]\end{array}$} & \multirow{2}{*}{$\begin{array}{c}\text { Modelo de } \\
\text { Regressor }\end{array}$} & \multicolumn{2}{|c}{ MAE $[m L / h]$ - Dispositivo Intravenoso } \\
\cline { 2 - 4 } & Infusão Gravitacional & Bombas de Infusão \\
\hline \multirow{2}{*}{250} & PMC & 43,2 & 18,6 \\
\cline { 2 - 4 } & LSTM & $\mathbf{1 3 , 2}$ & $\mathbf{0 , 9}$ \\
\hline \multirow{2}{*}{500} & PMC & 27,2 & 18,9 \\
\cline { 2 - 4 } & LSTM & $\mathbf{1 4 , 3}$ & $\mathbf{0 , 9}$ \\
\hline \multirow{2}{*}{1000} & PMC & 34,3 & 13,7 \\
\cline { 2 - 4 } & LSTM & $\mathbf{1 9 , 7}$ & 9,1 \\
\hline & PMC & 67,8 & $\mathbf{1 , 7}$ \\
\cline { 2 - 4 } & LSTM & $\mathbf{2 2 , 2}$ & \\
\hline
\end{tabular}

Considerando os resultados apresentados na Tabela 8, pode-se observar que o regressor com melhor inferência dos perfis de infusão é o LSTM, para ambos os dispositivos intravenosos. Como o LSTM alcançou os melhores resultados, as análises seguintes consideraram apenas este modelo.

Uma análise visual dos perfis inferidos de entrega de medicamentos intravenosos usando o modelo LSTM quando comparados com os valores simulados é apresentada nas Figuras 2 e 3. Estas figuras apresentam gráficos de barras e Curvas de Dispersão, usando perfis de liberação de medicamentos intravenosos, para ambas as infusões: gravitacional e com bombas.

Os gráficos de barras comparando os valores simulados e inferidos considerando as infusões gravitacionais são mostrados na Figura 2 parte (a) e considerando a bomba de infusão são mostrados na Figura 2 parte (b). Os eixos Y desses gráficos apresentam os valores da taxa de vazão e os eixos $\mathrm{X}$ apresentam o número de perfis de infusão.

Os resultados apresentados nestes dois gráficos ilustram que o regressor desenvolvido alcançou bons resultados ao modelar o comportamento da bomba de infusão, conforme apresentado na Figura 2 parte (b). Por outro lado, a modelagem para infusões gravitacionais atingiu um resultado um pouco pior, conforme apresentado na Figura 2 parte (a). Isso ocorre devido ao regime de líquido escoado, que caracteriza o comportamento do perfil de liberação do fármaco por infusões gravitacionais [Lovich and Peterfreund 2017].

As curvas de dispersão dos perfis do dispositivo de infusão por infusão gravitacional são mostradas na Figura 3 parte (a) e por bomba de infusão são mostradas na Figura 3 parte (b). A figura 3 partes (a) e (b) têm nos eixos Y o valor da taxa de infusões simulada e nos eixos $\mathrm{X}$ o valor da taxa de infusão inferida pelo regressor.

Ao observar-se as curvas de dispersão apresentadas na Figura 3, ficam mais evidenciadas as diferenças das inferências realizadas, considerando-se os dois dispositivos de infusão. Pode-se observar que os resultados das dispersões alcançados ao modelar as bombas de infusão, apresentados na Figura 3 (b), são mais precisos do que aqueles resultados alcançados ao modelar infusões gravitacionais, apresentados na Figura 3 (a). 


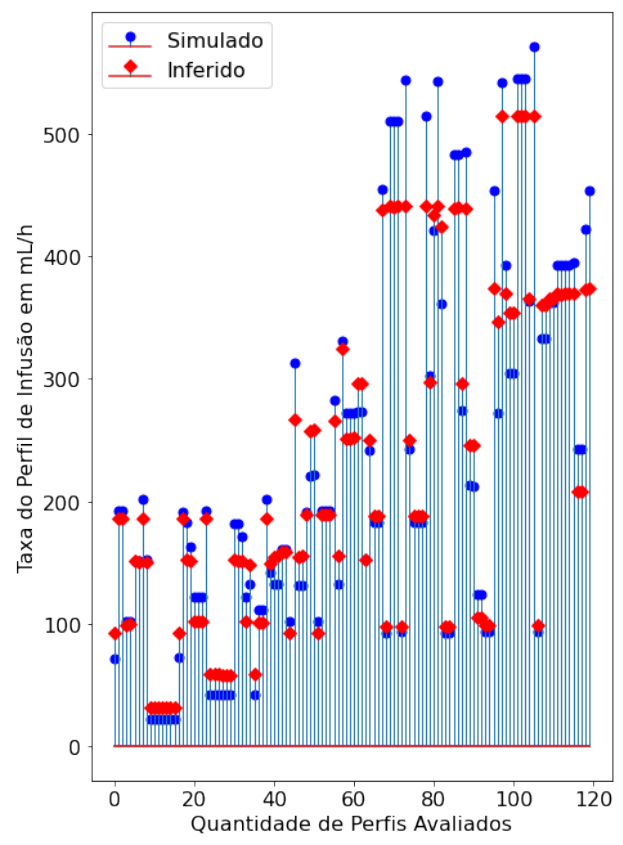

(a) Infusão Gravitacional

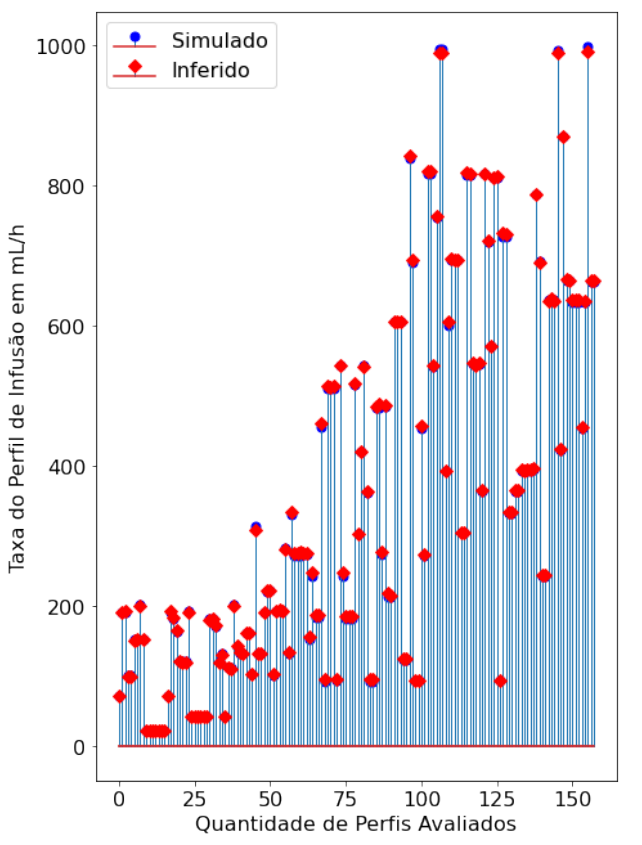

(b) Bomba de Infusão

Figura 2. Comparações das Inferências Geradas pelo Regressor LSTM.

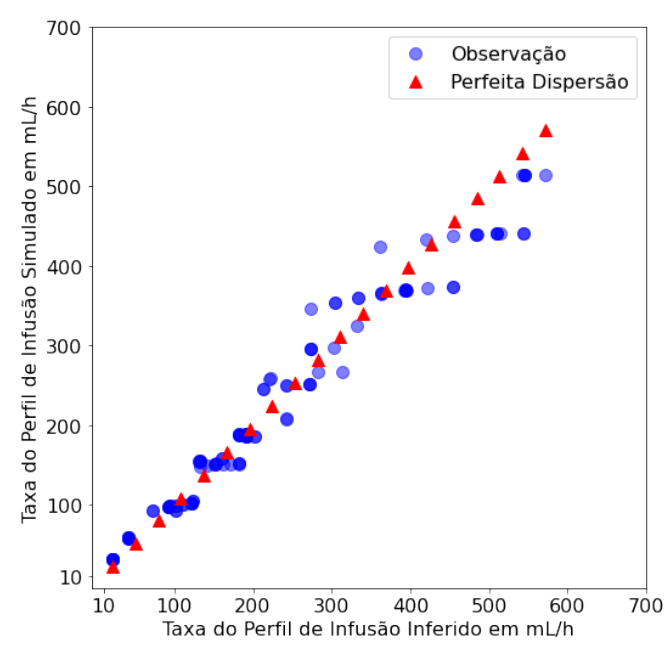

(a) Infusão Gravitacional

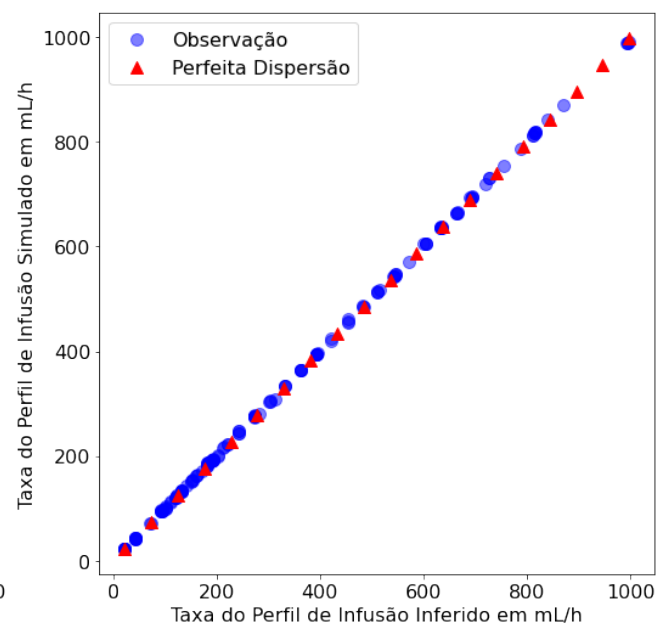

(b) Bomba de Infusão

Figura 3. Dispersões das Inferências Gerada pelo Regressor LSTM.

Pode-se observar, na Figura 3 (a) e na Figura 3 (b), as tendências das previsões quando comparadas com a linha da perfeita dispersão para os dois tipos de dispositivos de infusão. As análises visuais permitem observar, ainda, que os modelos de inferência para bombas de infusão alcançaram bons resultados para toda a faixa de vazão de 0 a $1000 \mathrm{~mL} / \mathrm{h}$. Já o modelo de infusão gravitacional alcançou bons resultados apenas para as taxas dos escoamentos menores que $200 \mathrm{~mL} / \mathrm{h}$.

Os resultados alcançados também foram avaliados por meio de Testes Estatísticos. O T-Test, disponível pela ferramenta SciPy, foi explorado [Virtanen and et al. 2020]. Este 
Teste considera a média de duas amostras independentes da estatística descritiva. É um teste bilateral para a hipótese nula de que duas amostras independentes têm valores médios idênticos [Feitosa Neto et al. 2019].

A ferramenta de biblioteca SciPy foi usada porque também é de código aberto. $\mathrm{O}$ teste estatístico usado é chamado de "ttest_ind_from_stats" nesta biblioteca. Para executar o T-Test, as médias e os desvios padrão das duas amostras são usados como entradas. Assim, é retornado o valor p_value, que assume, em hipótese nula, que as variações da amostra são iguais.

Na Tabela 9, os valores de probabilidades bicaudais da comparação entre os perfis de infusão simulados e inferidos são apresentados para os dois tipos de dispositivos de infusão intravenosa. Considerando estes resultados, da Tabela 9 para o p_value, é possível concluir com Intervalo de Confiança de $I C=95 \%$, que a hipótese nula, que define as distribuições com os mesmos valores, não pode ser negada, pois, todos os valores p_valor são maiores que 0,05 [Virtanen and et al. 2020].

A Tabela 9 também apresenta o índice do coeficiente de correlação de Pearson considerando os valores MAE dos perfis de infusão inferidos e simulados, organizados por seus volumes.

Os resultados da Tabela 9 mostraram que os modelos para infusões gravitacionais tiveram o pior desempenho. Os menores valores do coeficiente de correlação de Pearson e probabilidade para as inferências dos perfis de liberação do fármaco foram produzidos por infusão gravitacional, considerando os volumes de 125 e $500 \mathrm{~mL}$. Em volumes nominais com a menor quantidade de medicamento no reservatório, portanto, em decorrência da menor pressão hidrostática, reservatório de medicamento com menor quantidade de líquidos, estão sujeitos a uma menor ação da força de gravidade, devido à altura do líquido.

As variações típicas mostradas na Tabela 8 e na Figura 2 partes (a) e (b), estão dentro dos limites praticados para distribuição de medicamentos e também lidam com os requisitos dos padrões normativos para variações nas taxas de vazão para as entregas de medicamentos intravenosos [NBR 60601-2-24 2015].

Tabela 9. Análise de Correlação de Pearson e Probabilidade para o Modelo LSTM.

\begin{tabular}{c|c|c|c|c}
\hline \multirow{2}{*}{$\begin{array}{c}\text { Volume } \\
{[\mathrm{mL}]}\end{array}$} & \multicolumn{2}{|c|}{ Infusão Gravitacional } & \multicolumn{2}{c}{ Bombas de Infusão } \\
\cline { 2 - 5 } & Correlação & p_value & Correlação & p_value \\
\hline 125 & 0.876746 & 0.982197 & 0.999422 & 0.992866 \\
\hline 250 & 0.882168 & 0.981955 & 0.988709 & 0.995942 \\
\hline 500 & 0.955338 & 0.959878 & 0.991741 & 0.991867 \\
\hline 1000 & 0.957702 & 0.946022 & 0.999871 & 0.995504 \\
\hline
\end{tabular}

Assim, como mostrado na Tabela 8 e Tabela 9 e na Figura 2 e Figura 3, pode-se concluir que as inferências relacionadas às bombas de infusão tiveram um erro menor, do que as alcançadas para as infusões gravitacionais. Portanto, os resultados obtidos permitem verificar o potencial da modelagem MLP e LSTM, como método de apoio na implementação da automação de dispositivos de terapia por infusão. 


\subsection{Resultados da Avaliação Explorando o Conjunto de Dados Reais}

Nesta avaliação da aplicação explorando o conjunto de dados reais, que nunca foram manipulados nas fases de Treinamento e Teste, o objetivo é uma análise qualitativa dos resultados da inferência, utilizando o modelo de regressor LSTM. Lembrando que nesta aplicação com dados reais, o regressor LSTM já foi avaliado. Assim, essa análise caracteriza as alterações nos perfis de entrega dos medicamentos intravenosos, que serão disponibilizados aos profissionais de saúde.

Nesse sentido, a modelagem do regressor LSTM foi concebida a partir de dados simulados que compõem uma gama de combinações nas variações das taxas praticadas dos perfis das infusões intravenosas e das quantidades de medicamentos administradas para ambos os dispositivos médicos por infusões gravitacionais e por bombas.

Assim, na Figura 4, são apresentadas as identificações dos perfis reais das infusões, seja na parte (a) para Bomba de Infusão e na parte (b) para Infusão Gravitacional. Onde na Figura 4, o eixo $\mathrm{Y}$ indica os valores da velocidade das taxas de infusão em $m L / h$, o eixo X mostra a duração das infusões em horas e o escopo da Figura, as caixas de texto informam os valores dos perfis infusões e os volumes nominais de cada infusão intravenosa (rótulo Real) organizados por sequência numérica.

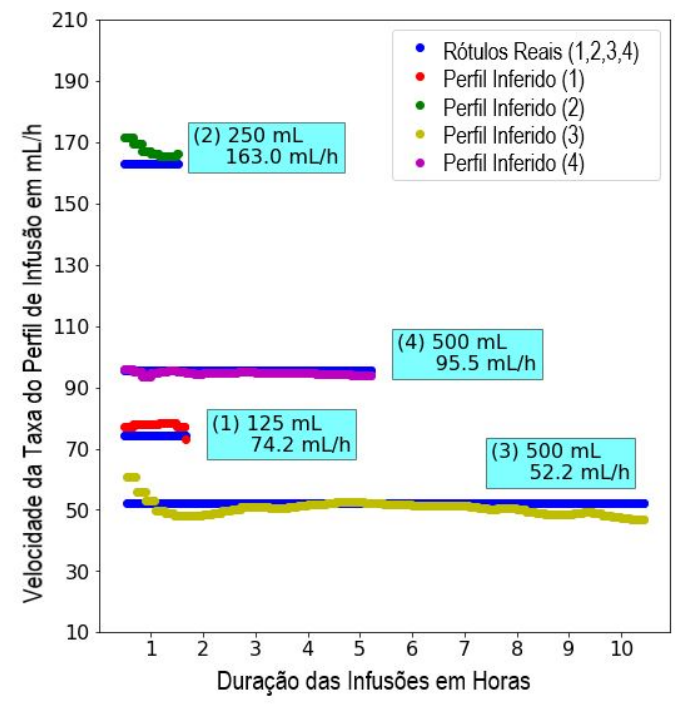

(a) Bomba de Infusão

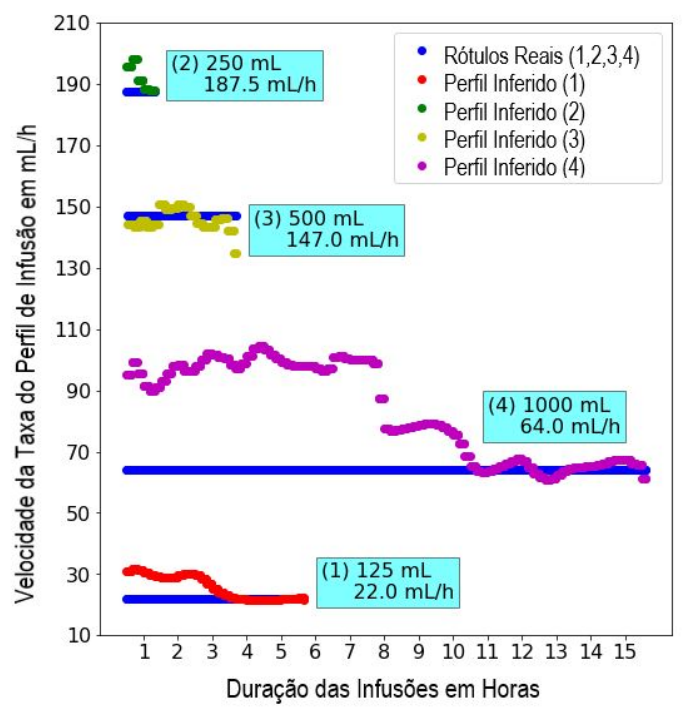

(b) Infusão Gravitacional

Figura 4. Avaliação do Estudo de Caso das Inferências dos Perfis de Infusões.

Considerando a Figura 4, podemos ver que as inferências das taxas de infusão da Bomba de Infusão tiveram um melhor desempenho com as inferências feitas por infusões gravitacionais; os resultados são esperados, considerando que as bombas de infusão têm como finalidade um melhor desempenho na administração de medicamentos intravenosos [Lovich and Peterfreund 2017].

Nesse sentido, os profissionais de saúde com acesso às informações sobre as infusões praticadas (Perfil Inferido), por exemplo, a ilustração da Figura 4, podem verificar a prescrição médica com o registro visual da infusão intravenosa realizada. Além disso, 
o próprio profissional médico pode questionar/verificar se sua prescrição para a infusão intravenosa manteve o curso durante a duração da medicação intravenosa.

\section{Conclusão}

Este artigo apresentou uma avaliação do desempenho do Aprendizado de Máquina na inferência de perfis de infusão intravenosa. Mais especificamente, o objetivo principal deste trabalho foi avaliar a utilização de dois métodos de regressão: MLP e LSTM. A perspectiva com os resultados obtidos é promover melhorias nos procedimentos de infusoterapia, que até o momento apresentam alta incidência de eventos adversos em seu funcionamento.

O acompanhamento e o registro dos perfis de infusão para administração de medicamentos intravenosos têm o potencial de reduzir os eventos adversos, uma vez que esses procedimentos no dia a dia do hospital ainda não são realizados sob inspeção automatizada. Então, uma solução capaz de descobrir e registrar de forma autônoma o volume e a taxa dos perfis de infusão pode contribuir para reduzir os eventos adversos e melhorar o tratamento do paciente.

A contribuição deste trabalho é introduzir um novo conceito na avaliação dos procedimentos de tratamento por infusoterapia, aprimorando e qualificando a tomada de decisão e os recursos tecnológicos dos profissionais de saúde em procedimentos de infusão intravenosa.

As avaliações dos modelos de inferência mostraram que o modelo de regressão LSTM foi o método que apresentou os melhores resultados para ambos os dispositivos de infusão intravenosa. Portanto, a análise estatística realizada permitiu concluir que os perfis inferidos são semelhantes aos simulados para ambos os dispositivos de infusão intravenosa.

Os resultados foram considerados promissores e apontam para a continuidade desta pesquisa. A expectativa é gerar novas abordagens para identificar perfis de infusão intravenosa, contribuindo para o desenho de sistemas de Engenharia Biomédica para monitoramento autônomo dos processos de liberação de medicamentos intravenosos.

\section{Referências}

Bhavasaar, M. K., Nithya, M., Praveena, R., Bhuvaneswari, N., and Kalaiselvi, T. (2016). Automated intravenous fluid monitoring and alerting system. In 2016 IEEE Technological Innovations in ICT for Agriculture and Rural Development (TIAR), pages 77-80, Chennai, India. IEEE.

Chambers, D. J. (2019). Principles of intravenous drug infusion. Anaesthesia \& Intensive Care Medicine, 20(1):61-64.

Dos Santos, H. D., Ulbrich, A. H. D., Woloszyn, V., and Vieira, R. (2019). DDC-Outlier: Preventing Medication Errors Using Unsupervised Learning. IEEE Journal of Biomedical and Health Informatics, 23(2):874-881.

ECRI, I. (2017). Top 10 Health Technology Hazards for Top 10 Health Technology Hazards for 2017. Technical report, ECRI Institute Patient Safety Organization, Massachusetts, USA, Massachusetts, USA. 
Feitosa Neto, A. A., Xavier, J. C., Canuto, A. M. P., and Oliveira, A. C. M. (2019). A comparative study on automatic model and hyper-parameter selection in classifier ensembles. In 2019 8th Brazilian Conference on Intelligent Systems (BRACIS), pages $323-328$.

Ferreira, F., Barbosa, J., Gruendemann, F., Machado, R., Yamin, A., and Agostini, L. (2019). Spodi: Simulador do perfil operacional de dispositivos intravenosos para auxilio à tomada de decisões médicas. In Anais do XIX Simpósio Brasileiro de Computação Aplicada à Saúde, pages 34-45, Porto Alegre, RS, Brasil. SBC.

Fink, D., Wagner, A., and Ehlers, W. (2018). Application-driven model reduction for the simulation of therapeutic infusion processes in multi-component brain tissue. Journal of Computational Science, 24:101-115.

Hao, J. and Ho, T. K. (2019). Machine Learning Made Easy: A Review of Scikit-learn Package in Python Programming Language. Journal of Educational and Behavioral Statistics, 44(3):348-361.

Lovich, M. A. and Peterfreund, R. A. (2017). Drug Flow Through Clinical Infusion Systems: How Modeling of the Common-volume Helps Explain Clinical Events. Pharmaceutical Technology in Hospital Pharmacy, 2(2):49-62.

NBR 60601-2-24, A. B. d. N. T. (2015). Equipamento eletromédico Parte 2-24: Requisitos particulares para a segurança básica e o desempenho essencial de bombas de infusão e de controladores de infusão. $A B N T$.

NEITZKE F., F. (2020a). Github - ffabricio/medical-device: Intravenous drug delivery devices. https://github.com/FFabricio/ Data-Set-for-Intravenous-Infusion-Profiles-.git. (Acessado em 07/08/2020).

NEITZKE F., F. (2020b). Infusionprofiler.ipynb - colaboratory. https://colab.research.google.com/drive/ 1ZOrrrMci4asHoEICX5nMgMmLJn8TAw5j?usp=sharing. (Acessado em 07/01/2021).

Paszke, A., Gross, S., Massa, F., Lerer, A., Bradbury, J., Chanan, G., Killeen, T., Lin, Z., Gimelshein, N., Antiga, L., Desmaison, A., Kopf, A., Yang, E., DeVito, Z., Raison, M., Tejani, A., Chilamkurthy, S., Steiner, B., Fang, L., Bai, J., and Chintala, S. (2019). Pytorch: An imperative style, high-performance deep learning library. In Advances in Neural Information Processing Systems 32, pages 8024-8035. Curran Associates, Inc.

Pedregosa, F., Varoquaux, G., Gramfort, A., Michel, V., Thirion, B., Grisel, O., Blondel, M., Prettenhofer, P., Weiss, R., Dubourg, V., Vanderplas, J., Passos, A., Cournapeau, D., Brucher, M., Perrot, M., and Duchesnay, E. (2011). Scikit-learn: Machine learning in Python. Journal of Machine Learning Research, 12:2825-2830.

Sait, M. K., Aguam, A. P., Mohidin, S., Eidraous, S. A., Tabsh, L. A., Anfinan, N. M., Khalili, B. M. A., Sait, H. K., and Sait, K. H. (2019). Intravenous Site Complications for Patients Receiving Chemotherapy: An Observational Study. Annals of Short Reports, 2(1032):1-4.

Virtanen, P. and et al. (2020). SciPy 1.0: Fundamental Algorithms for Scientific Computing in Python. Nature Methods, 17:261-272. 